\title{
A perfected device for performing retrograde urethrography
}

\author{
Juan D Berná-Mestre Jr' \\ JD Berná-Serna²
}

'Department of Radiology, University Hospital of Salamanca, Spain; ${ }^{2}$ Department of Radiology, Virgen de la Arrixaca University Hospital,

$30 I 20$ El Palmar (Murcia), Spain
Correspondence: Juan D Berná-Mestre Jr Departamento de Radiología, Hospital Universitario de Salamanca, Paseo San Vicente s/n, 37007, Salamanca, Spain Tel +34 92329 II 153

Fax +34 92329 II 3 I

Email mesjubermu@hotmail.com

\begin{abstract}
The present study describes a perfected device for retrograde urethrography (RUG). The device allows RUG to be performed using a balloonless catheter and with assessment of the various problems of the urethra. The procedure is simple and well tolerated by patients and represents an interesting alternative to the conventional RUG technique.
\end{abstract}

Keywords: urethra, retrograde urethrography, urethral stricture, device

Retrograde urethrography (RUG) is considered to be the best initial study for urethral and periurethral imaging in men. Currently the most widely used RUG technique involves a Foley catheter (conventional technique). Fixation of the catheter is achieved by inflating a balloon in the fossa navicularis, which prevents extravasation on introduction of contrast material. No local anesthesia is used, nor is the use of lubrication recommended, such that the patient notes discomfort on introduction of the catheter and inflation of the balloon. Moreover, this technique cannot be performed in cases of stenosis, malformations or previous surgery in the urethromeatal area (Pavlica et al 2003; Kawashima et al 2004).

In a previous publication (Berná et al 2003) we described a clamp device for performing RUG and showed it to be a good alternative to the conventional technique. The present study describes a perfected clamp device, which together with a prelubricated catheter allows RUG to be performed simply.

Two parts have been incorporated into the model described in 2003: a catheter guide and a padded ring to prevent contact between the adjustable band and the skin; the base of the device has also been modified. A 10-Fr EasyCath prelubricated catheter (Colopast) is coupled to the device but a 6-Fr catheter is used in the event of stenosis (Figure 1). The foreskin, if present, is retracted to permit sterile preparation of the glans penis with betadine. After purging, the catheter is inserted into the urethral meatus and the device moved in a proximal direction until the padded ring surrounds the crown of the glans. The clamp is tractioned and external compression applied, and a syringe is connected to the catheter to inject contrast material. After checking that there is no extravasation of contrast material the RUG is performed (Figure 2). Once the technique is finished the band is cut at the V-notch for decompression so that the device and catheter can be removed easily in one piece. We believe this simple method may help increase the success of performing RUG in men. The discomfort of the conventional technique is reduced, as a prelubricated catheter is used and no balloon needs to be inflated; extravasation of contrast material is avoided by external compression and the catheter is held in place by a guide. RUG can also be performed in the case of alterations in the urethromeatal area (stenosis, meatotomy, hypospadias, etc.) when the conventional technique is difficult or impossible to perform. 


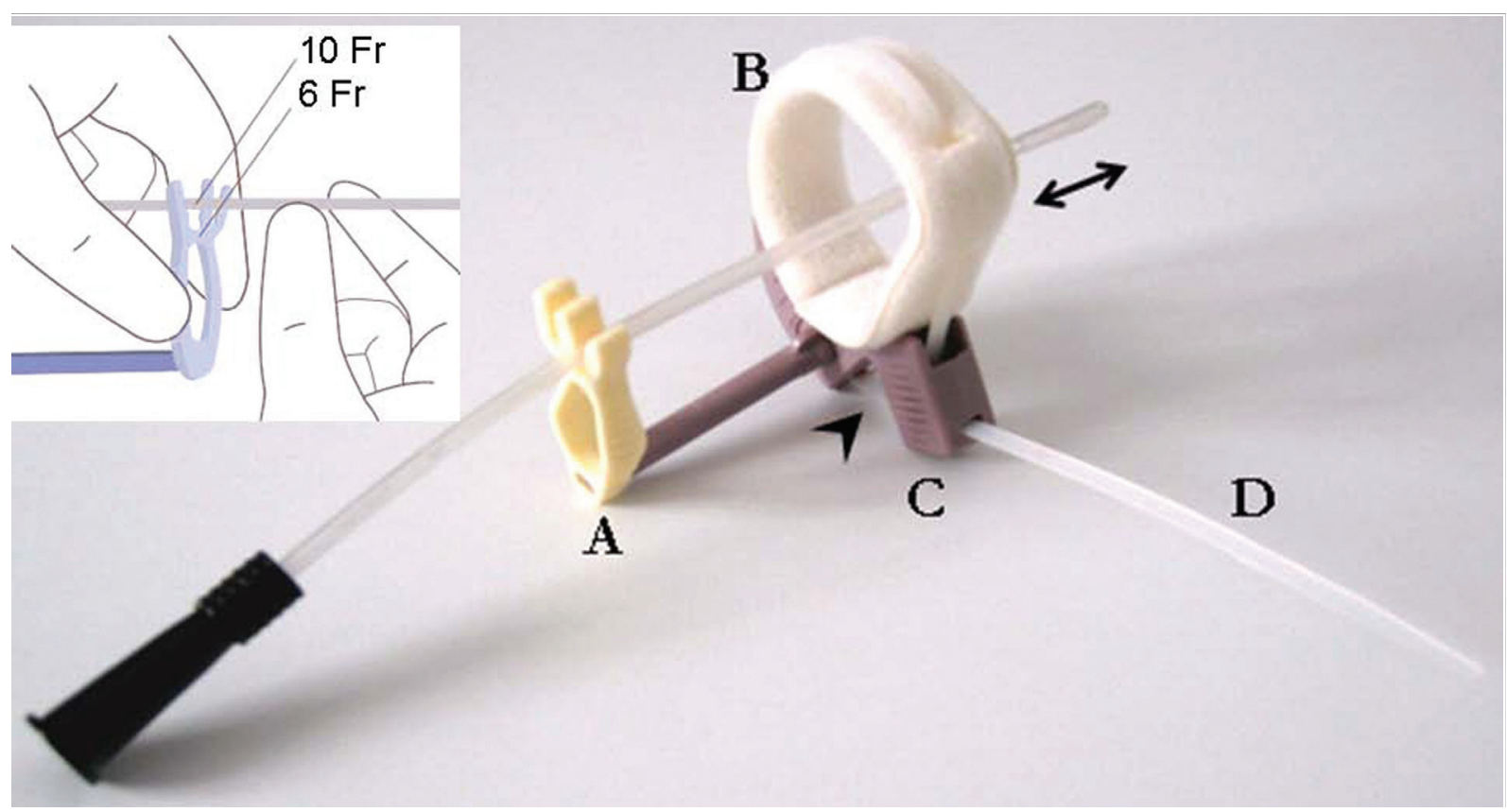

Figure I Illustration of the device coupled to a catheter. A) Catheter guide: the guide has two channels to fit 6- and I0-Fr catheters. B) Padded ring: the perforated tip of the catheter must be pushed beyond the ring (double-headed arrow) so that the contrast material can flow unobstructed after external compression. C) Base: incorporates a V-notch (arrowhead) to enable the band to be cut for decompression. D) Adjustable band: the band is tractioned to achieve external compression.

A

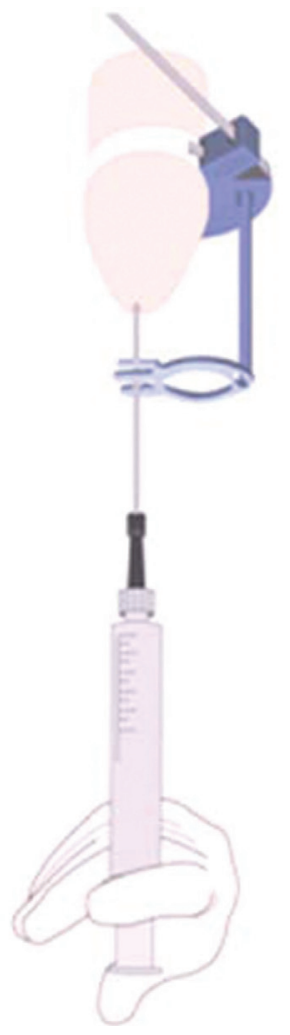

B

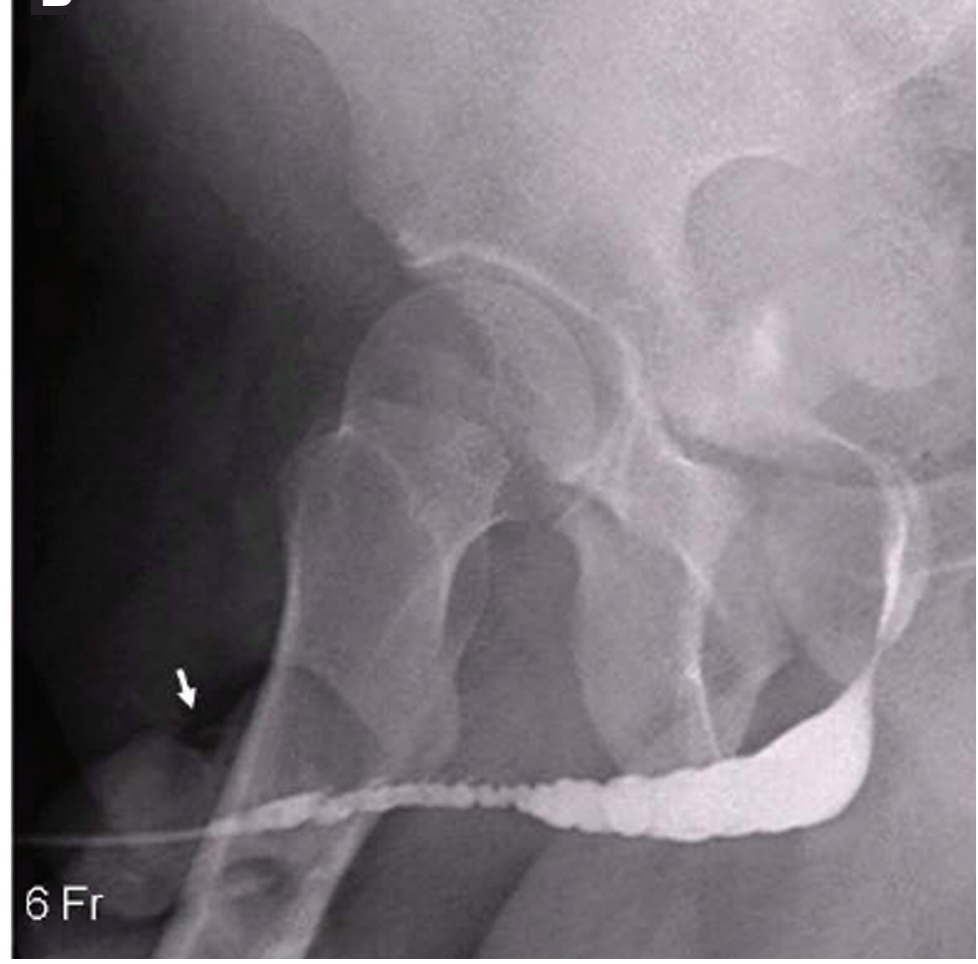

Figure 2 Technique for retrograde urethrography. A) Illustration showing injection of contrast material with a syringe connected to the catheter and the device fitted to the penis. B) Retrograde urethrogram showing stenosis of the penile urethra. Observe the external compression (arrow) achieved with the device. 
At our hospital we used routinely clamp device for performing RUG. At present we are using a sterile, single-use device. The procedure described is simple, rapid, reliable, and well tolerated by patients.

\section{Disclosure}

The authors report no conflicts of interest in this work.

\section{References}

Berná JD, Berná JD Jr. 2003. A new device for retrograde urethrography in the male. Eur Radio, 13:1420-2.

Kawashima A, Sandler CM, Wasserman NF, et al. 2004. Imaging of Urethral Disease: A Pictorial Review. RadioGraphics, 24:195-216.

Pavlica P, Barozzi L, Menchi I. 2003. Imaging of male urethra. Eur Radiol, 13:1583-96. 
